\title{
Neuromodulation of $I_{\mathrm{h}}$ in Layer II Medial Entorhinal Cortex Stellate Cells: A Voltage-Clamp Study
}

\author{
James G. Heys and Michael E. Hasselmo \\ Center for Memory and Brain, Graduate Program for Neuroscience, Department of Psychology, Boston University, Boston, Massachusetts 02215
}

Stellate cells in layer II of medial entorhinal cortex $(\mathrm{mEC})$ are endowed with a large hyperpolarization-activated cation current [h current $\left(I_{\mathrm{h}}\right)$ ]. Recent work using in vivo recordings from awake behaving rodents demonstrate that $I_{\mathrm{h}}$ plays a significant role in regulating the characteristic spatial periodicity of "grid cells" in mEC. A separate, yet related, line of research demonstrates that grid field spacing changes as a function of behavioral context. To understand the neural mechanism or mechanisms that could be underlying these changes in grid spacing, we have conducted voltage-clamp recordings of $I_{\mathrm{h}}$ in layer II stellate cells. In particular, we have studied $I_{\mathrm{h}}$ under the influence of several neuromodulators. The results demonstrate that $I_{\mathrm{h}}$ amplitude can be both upregulated and downregulated through activation of distinct neuromodulators in mEC. Activation of muscarinic acetylcholine receptors produces a significant decrease in the $I_{\mathrm{h}}$ tail current and a hyperpolarizing shift in the activation, whereas upregulation of cAMP through application of forskolin produces a significant increase in the $I_{\mathrm{h}}$ amplitude and a depolarizing shift in $I_{\mathrm{h}}$ activation curve. In addition, there was evidence of differential modulation of $I_{\mathrm{h}}$ along the dorsal-ventral axis of mEC. Voltage-clamp protocols were also used to determine whether M current is present in stellate cells. In contrast to CA1 pyramidal neurons, which express M current, the data demonstrate that M current is not present in stellate cells. The results from this study provide key insights into a potential mechanism that could be underlying changes seen in grid field spacing during distinct behavioral contexts.

\section{Introduction}

Characterizing the unique electrophysiological profile of distinct neuronal subtypes remains a central problem in neuroscience research. Understanding neuromodulation of ion channels is a problem of particular complexity because of the possibility that the modulation of any one of several biochemical pathways, or the modulation of a combination of such biochemical pathways, could influence the activity of a single ion channel. Using wholecell voltage-clamp recordings of stellate cells (SCs) in layer II of medial entorhinal cortex ( $\mathrm{mEC}$ ), we have focused attention upon neuromodulation of hyperpolarization-activated cation current [h current $\left.\left(I_{\mathrm{h}}\right)\right] . I_{\mathrm{h}}$ is of particular interest in SCs because of its large amplitude and potential role in membrane potential rhythmicity (Dickson et al., 2000) and resonance (Haas and White, 2002; Erchova et al., 2004; Giocomo et al., 2007), as well as its putative role in the characteristic spatial representation shown by entorhinal neurons during extracellular recordings from awake behaving rats and mice (Giocomo et al., 2011). Unit recording studies have demonstrated that neurons in entorhinal cortex, termed grid cells, fire selectively at regular spatial intervals as the animal explores a 2D environment (Fyhn et al., 2004; Hafting et al., 2005; Moser and Moser, 2008). Together, the multiple firing

\footnotetext{
Received Feb. 22, 2012; revised April 19, 2012; accepted May 12, 2012.

Author contributions: J.G.H. and M.E.H. designed research; J.G.H. performed research; J.G.H. analyzed data;

J.G.H. and M.E.H. wrote the paper.

We thank A. Bogaard for programming assistance and L. Giocomo and N. Spruston for useful advice.

Correspondence should be addressed to James G. Heys, Graduate Student, Graduate Program for Neuroscience,

Boston University, 2 Cummington St., Boston, MA 02139. E-mail: jimheys@bu.edu.

DOI:10.1523/JNEUROSCI.0868-12.2012

Copyright $\odot 2012$ the authors $\quad 0270-6474 / 12 / 329066-07 \$ 15.00 / 0$
}

fields of a single grid cell form a nearly symmetrical hexagonal grid, tiling the floor of the animal's environment. Previous work has shown that not only does $I_{\mathrm{h}}$ physiology correlate with grid field spacing and grid field size (Giocomo et al., 2007), but recently it has been demonstrated that knock-out of the HCN1 subunit can alter the grid field spacing and grid field size (Giocomo et al., 2008, 2011). In addition to its functional role in the mEC SCs, $I_{\mathrm{h}}$ has also been shown to be particularly disposed to influences by different modulatory systems (DiFrancesco and Tortora, 1991; Pian et al., 2006, 2007; Rosenkranz and Johnston, 2006; Ma et al., 2007).

This work is also inspired by our previous research demonstrating cholinergic modulation of membrane potential resonance in mEC SCs (Heys et al., 2010). Similar to $I_{\mathrm{h}}$ properties that correlate to grid field spacing and grid field size, membrane potential resonance has also been found to correlate with grid spacing (Giocomo et al., 2007). The results of our previous study suggested that the decrease in membrane potential resonance frequency and resonance strength, following application of cholinergic agonists, was at least in part the result of changes in $I_{\mathrm{h}}$. We have extended this result in the current study through pharmacological isolation of $I_{\mathrm{h}}$ in voltage clamp. The role of h current is further supported here by our voltage-clamp recordings showing that M current $\left(I_{\mathrm{m}}\right)$ is not significantly present in stellate cells. This result is in contrast to hippocampal pyramidal neurons, where $I_{\mathrm{m}}$ is present and responsible for membrane potential resonance at more depolarized resting potentials (Hu et al., 2002). In addition to resolving previous claims that $I_{\mathrm{m}}$ could be present in SCs and responsible for membrane potential oscillations, this result further exemplifies the distinct electrophysiological profile 
of SCs relative to other principal excitatory neurons in the parahippocampal region.

\section{Materials and Methods}

Slice preparation. Tissue analyzed in this study was taken from 17-21-dold male and female Long-Evans rats (Charles River Laboratories). The Institutional Animal Care and Use Committee at Boston University approved all experimental techniques. Animals were deeply anesthetized with isoflurane (Abbott Laboratories) and decapitated. The brain was rapidly removed and placed in oxygenated $4^{\circ} \mathrm{C}$ ACSF with the following concentrations (in mM): $125 \mathrm{NaCl}, 2.0 \mathrm{CaCl}, 2.5 \mathrm{KCl}, 1.25 \mathrm{NaH}_{2} \mathrm{PO}_{4}, 25$ $\mathrm{NaHCO} 3,25 \mathrm{D}$-glucose, and $1.0 \mathrm{MgCl}$. The brain was then blocked and glued to a platform and a vibratome (Leica VT1000s) was used to section $400 \mu \mathrm{m}$ horizontal slices of the parahippocampal formation. Slices were cut in succession from dorsal to ventral. Previous work demonstrated that slice direction does not alter cellular physiology (Giocomo et al., 2007). After the slicing procedure, the tissue was incubated for $30 \mathrm{~min}$ in a heat bath set to $31^{\circ} \mathrm{C}$ and incubated at room temperature for $30 \mathrm{~min}$.

Electrophysiological recordings. Slices were placed in a 20 Series infusion chamber while maintaining bath temperature at $37^{\circ} \mathrm{C}$ (Warner Instruments) and slices were infused with oxygenated ACSF. A P-87 pipette puller (Sutter Instruments) was used to make $2-5 \mathrm{M} \Omega$ borosilicate glass capillary tubes. Whole-cell patch-clamp recordings were made in voltage clamp and current clamp. Briefly, the electrode resistance was monitored using an oscilloscope and brief pulses of inward pressure were used to obtain whole-cell recordings once a "GIGA seal" was formed. Series resistance was monitored over the course of each session and only recordings that maintained a series resistance of $<10 \mathrm{M} \Omega$ were included in the study. Pipettes were loaded with intracellular solution containing (in $\mathrm{mM}$ ) the following: $120 \mathrm{~K}$-gluconate, 10 HEPES, 0.2 EGTA, $20 \mathrm{KCL}, 2 \mathrm{MgCl}, 7$ diTrisPhCr, $4 \mathrm{Na}_{2} \mathrm{ATP}$, and 0.3 TrisGTP (pH adjusted to 7.3 with $\mathrm{KOH}$ ). Recordings were made using a Multiclamp 700B amplifier (Molecular Devices). The data were low-pass filtered at $10 \mathrm{kHz}$ and digitized using Digidata 1320 at a sampling frequency of 20 $\mathrm{kHz}$. Recorded signals were collected on a Pentium-based computer running Clampex 10.0 recording software (Molecular Devices). Liquid junction potential was measured to be between 5 and $6 \mathrm{mV}$ using the technique of Neher (1992) and Dickson et al. (2000). All recordings are not corrected for the liquid junction potential.

Stellate cells were identified visually in the slice by laminar location, cellular morphology, and through confirmation of their characteristic electrophysiological profile: large amplitude sag potential, the presence of large amplitude membrane potential oscillations, spike clustering, and membrane potential resonance of 3-15 Hz. Cells had an input resistance $\left(R_{\text {in }}\right)$ of $25-80 \mathrm{M} \Omega$ and a resting membrane potential $\left(V_{\mathrm{r}}\right)$ of -58 to -65 $\mathrm{mV}$. Data are expressed as mean \pm SEM.

Solutions. All recording solutions of ACSF were bath applied and included $1 \mu \mathrm{M}$ TTX to block voltage-sensitive sodium currents, $2 \mathrm{~mm} \mathrm{CoCl} 2$ to block voltage-sensitive calcium currents, and $20 \mathrm{~mm}$ tetraethylammonium to block voltage-sensitive potassium channels. Where stated, some solutions also included other agents, including $10 \mu \mathrm{M}$ carbachol (CCh), $1 \mu \mathrm{M}$ atropine, $10 \mu \mathrm{M}$ XE991, $50 \mu \mathrm{M}$ forskolin, or $100 \mu \mathrm{M}$ ZD7288.

Data analysis. All data were recorded using P-Clamp 10.0 (Molecular Devices) and analyzed using Matlab. All statistical comparisons between group averages were made using the Kruskal-Wallis nonparametric oneway ANOVA to avoid assumptions of normality. h Current was activated in voltage clamp using $3 \mathrm{~s}$ duration hyperpolarizing voltage steps. Tail currents were produced by these voltage steps starting from and returning to a holding potential of $-40 \mathrm{mV}$ and stepping to hyperpolarized holding potentials ranging in $10 \mathrm{mV}$ increments from -120 to $-50 \mathrm{mV}$. h Current amplitudes were measured as the difference between the instantaneous tail current response and the steady-state tail current response. h Current steady-state activation curves were measured by fitting the difference between instantaneous tail current and steady-state tail current amplitude responses to Boltzman equations of the following form:

$$
I(V)=\frac{1}{1+e^{\left(\frac{V-V_{1 / 2}}{k}\right)}}
$$

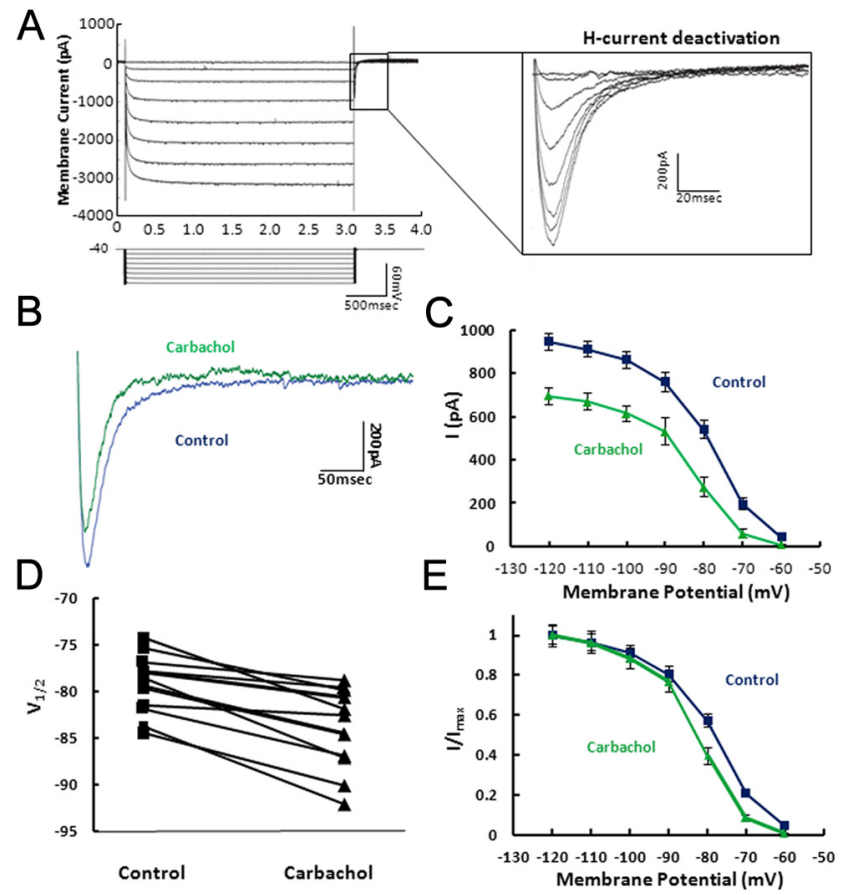

Figure 1. Analysis of $/_{\mathrm{h}}$ tail current magnitude in the presence and absence of carbachol. $A, I_{\mathrm{h}}$ tail currents were obtained using 3 s hyperpolarizing voltage steps starting from and returning to a holding potential of $-40 \mathrm{mV}$. Inset, For each voltage step used to activate $I_{h}$, the tail current was measured as the difference between the minimum point of the tail current- - that is the minimum point of the tail current observed after the step back to $-40 \mathrm{mV}$ - and the steady-state current value. $\boldsymbol{B}$, Individual example tail currents are shown in control (blue) and after $10 \mathrm{~min}$ bath application of $10 \mu \mathrm{m}$ carbachol (green). C, Population averages demonstrate that carbachol significantly decreases the tail current measured at $-40 \mathrm{mV}$ after stepping from a series of holding potentials $(-120$ to $-50 \mathrm{mV})$. $D, V_{1 / 2}$ values, generated using the fits in Figure $1 D$, are measured across individual cells for control (square) and for carbachol (triangle).E, Tail current activation curves obtained in control (blue square) and in the presence of carbachol (green triangle) are shown normalized to maximum current.

where $I$ is the normalized steady-state membrane current at membrane potential $V, V_{1 / 2}$ is the membrane potential that gives rise to the half maximal current response, and $k$ is the slope of the steady-state activation curve. h Current activation time constants were measured by fitting the membrane tail current responses to single or double exponential equations.

Subthreshold membrane potential resonance was measured according to Heys et al. (2010). In current clamp, the membrane potential was recorded in response to an injection of a constant amplitude sinusoidal current stimulus that increased linearly from 0 to $20 \mathrm{~Hz}$ over $20 \mathrm{~s}$. The impedance profile was measured by taking the fast Fourier transform of the voltage response divided by the fast Fourier transform of the injected current, and the resonance frequency was defined as the frequency of current stimulation at the peak of the impedance profile.

\section{Results}

Previous research from single-cell recordings using the in vitro slice preparation have demonstrated that $I_{\mathrm{h}}$ plays an important role in the function of mEC SCs (Alonso and Llinas, 1989; Dickson et al., 2000; Giocomo and Hasselmo, 2008, 2009) that may influence properties of neurons tested by unit recordings in awake behaving animals (Hafting et al., 2005; Giocomo et al., 2011). It is also clear from previous work that it is possible to modulate $I_{\mathrm{h}}$ through regulation of several distinct neuromodulatory systems (Rosenkranz and Johnston, 2006; Ma et al., 2007; Pian et al., 2007). However, since h channel composition is a function of the expression of several possible combinations of HCN (hyperpolarization-activated/cyclic nucleotide) subunits and regulatory subunits, it is not possible to predict the effect of 
each modulatory system across different cell types in distinct brain regions. To this end, we have used whole-cell patch-clamp recordings of SCs in the slice preparation and isolated $I_{\mathrm{h}}$ in voltage clamp to study the effects of activating specific neuromodulatory pathways. From an initial holding potential of $-40 \mathrm{mV}$, cells were hyperpolarized to potentials that activate $I_{\mathrm{h}}$ (Fig. 1A). h Current amplitude was measured by comparing the difference between the instantaneous (peak) tail current amplitude and the steady-state current amplitude (Fig. 1A, inset). After bath application of the muscarinic acetylcholine receptor agonist CCh $(10 \mu \mathrm{M})$, there was a significant decrease in the $h$ current amplitude (Fig. $1 B$ ) as shown by a decrease in the instantaneous (peak) tail current. The population averages show that the mean maximum tail current amplitude, as measured after a step from a hyperpolarized holding potential of -120 $\mathrm{mV}$ to a holding potential of $-40 \mathrm{mV}$, decreased by $250.97 \pm 24.18 \mathrm{pA}$ after application of CCh $(p<0.01, n=25)$ (Fig. 1C). CCh produced a shift in the mean $V_{1 / 2}$ of the activation curve shifts from $-79.28 \pm 0.63 \mathrm{mV}$ in control ACSF to $-84.05 \pm 0.61 \mathrm{mV}$ in CCh $(p<0.01, n=25)$ (Fig. $1 D)$ and produced a leftward shift in the normalized activation curve of $I_{\mathrm{h}}$ (Fig. $1 E$ ).

One of the primary concerns with whole-cell patch-clamp experiments is the potential appearance of electrophysiological artifacts from biochemical washout induced by whole-cell patchclamp dialysis. To control for this possible problem, a separate set of SCs were patch clamped for 1.5 times the length of the period used for drug wash experiments (15 min total). $I_{\mathrm{h}}$ tail current amplitudes were measured in control ACSF before the delay and in the same control ACSF after the 15 min wait period, which is referred to as the time control condition. The $I_{\mathrm{h}}$ tail current measured at steps from -120 to $-40 \mathrm{mV}$ in control ACSF before the delay was $1036.5 \pm 49.8 \mathrm{pA}$. In control ACSF after the time control period, the $I_{\mathrm{h}}$ tail current was $1025.7 \pm 48.1 \mathrm{pA}(p=0.8728$, $n=6)(2 A, B)$. In addition to the lack of a change in the tail current measured at $-120 \mathrm{mV}$, there were no significant changes in the tail current amplitude measured after steps returning from other potentials ranging from -50 to $-120 \mathrm{mV}$ (data not shown). To ensure CCh-dependent modulation of $I_{\mathrm{h}}$ is acting through activation of mAChRs, tail currents were measured in 1 $\mu \mathrm{M}$ atropine, a competitive $\mathrm{mAChR}$ antagonist and measured after subsequent application of $1 \mu \mathrm{M}$ atropine with $10 \mu \mathrm{M}$ CCh. No significant change was observed after application of CCh with atropine, suggesting that the cholinergic effects shown in Figure 1 are in fact acting through mAChRs $(2 C, D)$. The average $I_{\mathrm{h}}$ tail current amplitude measured after steps from a hyperpolarized holding potential of $-120 \mathrm{mV}$ to a holding potential of $-40 \mathrm{mV}$ in atropine was $945.0 \pm 71.8 \mathrm{pA}$. After application of $10 \mu \mathrm{M} \mathrm{CCh}$ with atropine, the $I_{\mathrm{h}}$ tail current was $927.7 \pm 63.9 \mathrm{pA}$ ( $p=0.564$, $n=4)(2 A, B)$. In addition to the lack of a change of the tail current measured after steps from $-120 \mathrm{mV}$, there were no significant changes in the tail current amplitude measured after steps returning from other potentials ranging from -50 to -120 $\mathrm{mV}$ (data not shown). To ensure that the current measured throughout this study is solely $I_{\mathrm{h}}$, tail currents were measured in control and after application of $100 \mu \mathrm{M}$ ZD7288. The tail current was completely eliminated after application of ZD7288 (Fig. 2E). The tail current was measured to be $859.4 \pm 114.8 \mathrm{pA}$ in control conditions and $0.8 \pm 11.7 \mathrm{pA}$ after application of ZD7288 $(p<$ $0.01 ; n=4)$ (Fig. 2F).

Seminal work from DiFrancesco and Tortora (1991) demonstrated that the voltage dependence of $I_{\mathrm{h}}$ activation, expressed in sinoatrial node myocytes, can be significantly altered though modulation of cyclic nucleotides. As such, we tested whether increasing cAMP levels through application of forskolin could also modulate $I_{\mathrm{h}}$ in SCs of entorhinal cortex. Bath application of 50 $\mu \mathrm{M}$ forskolin caused a significant increase in the $I_{\mathrm{h}}$ tail current (Fig. 3A). From the tail current measured at the maximum activation potential of $-120 \mathrm{mV}, I_{\mathrm{h}}$ amplitude increased by $109.0 \pm$ $19.1 \mathrm{pA}$ after application of forskolin $(p<0.05, n=10)$ as shown in Figure $3 B$. The Boltzman fits for the steady-state activation curves demonstrate that cAMP causes a shift in the $V_{1 / 2}$ from $-77.98 \pm 0.92 \mathrm{mV}$ in control ACSF to $-71.5 \pm 0.61 \mathrm{mV}$ after application of forskolin (Fig. $3 C$ ) and a rightward shift in the activation curve $(p<0.05, n=10)$ (Fig. 3D).

Previous work has suggested that the M current may be responsible for subthreshold membrane potential oscillations in SCs and membrane potential resonance at more depolarized potentials (Yoshida and Alonso, 2007; Heys et al., 2010). As such, we sought to provide the first direct measurement of $\mathrm{M}$ current in mEC SCs in voltage clamp. Surprisingly, when using the standard deactivation protocol to identify $\mathrm{M}$ current, we found no evidence to suggest $\mathrm{M}$ current is expressed in SCs (Fig. 4A). Compared with recordings in control ACSF, application of the selective M current blocker XE911 does not produce a significant change in the membrane current generated during a step from -30 to $-55 \mathrm{mV}(p=0.81, n=5)$ (Fig. $4 B)$. This is in contrast to subsequent application of $\mathrm{h}$ current blocker ZD7288, which completely eliminates the rectifying current generated in the control and XE991 condition during the step from -30 to $-55 \mathrm{mV}$ ( $p<$ $0.01, n=5)$ (Fig. $4 A, B$ ). It has been previously demonstrated that $\mathrm{M}$ current is present in pyramidal neurons in the CA1 region of the hippocampus (Halliwell and Adams, 1982; Storm, 1990). As a 
A
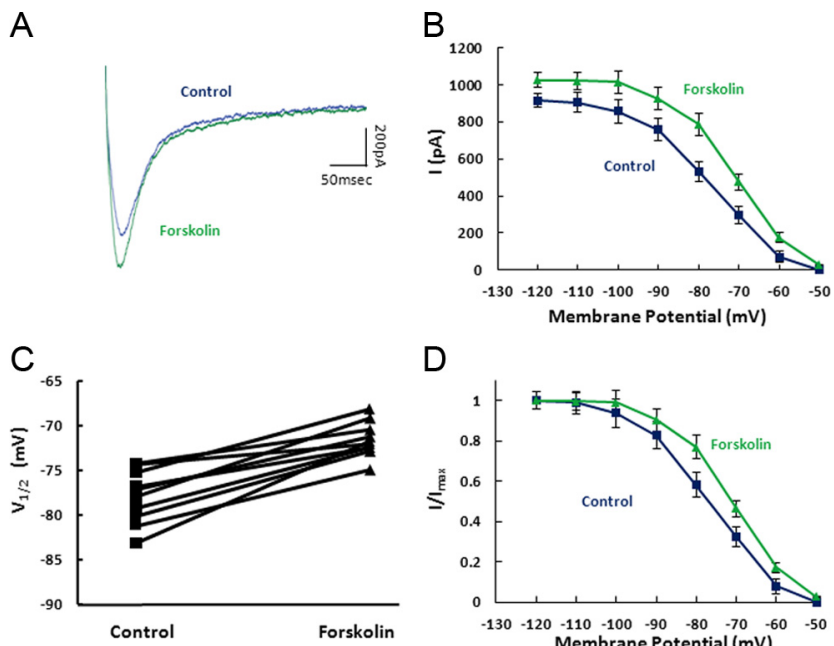

D

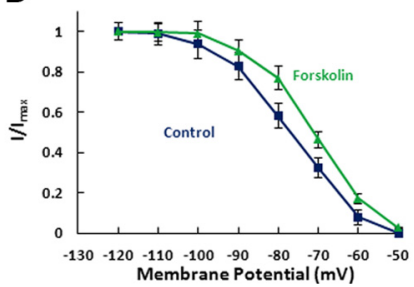

Figure 3. cAMP modulation of $I_{\mathrm{h}}$ tail current. $\boldsymbol{A}$, Individual tail currents were measured in control (blue) and after $10 \mathrm{~min}$ bath application of $50 \mu \mathrm{m}$ forskolin (green). B, Population averages demonstrate that forskolin significantly increases the tail current measured at -40 $\mathrm{mV}$ after stepping from a series of holding potentials $(-120$ to $-50 \mathrm{mV})$. C, The $V_{1 / 2}$ values, generated from Boltzman fits to the normalized activation curves, are measured across individual cells for control (square) and for forskolin (triangle). D, Tail current activation curves obtained in control (blue square) and in the presence of forskolin (green triangle) are shown normalized to maximum current.
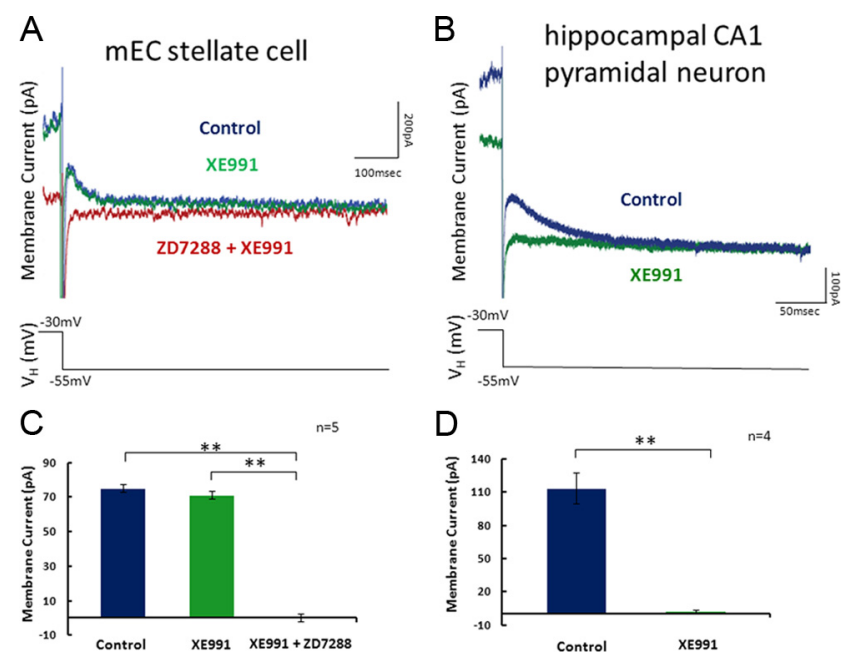

Figure 4. Analysis of $I_{M}$ in stellate neurons and $C A 1$ pyramidal neurons in the hippocampus. $A$, Whole-cell patch-clamp recordings were made in $\mathrm{mEC}$ layer II stellate cells. Tail currents measured during voltage steps to $-55 \mathrm{mV}$ from a holding potential of $-30 \mathrm{mV}$ in control ACSF (blue), after application of XE991 (green), and finally after application of XE991 with ZD7288 (red). $\boldsymbol{B}$, Population averages demonstrate that there is no significant change in the membrane current response between control ACSF and XE991. After subsequent application of ZD7288, the rectifying membrane current is completely abolished. C, Whole-cell patch recordings were made in hippocampal CA1 pyramidal neurons. Tail currents were measured in control ACSF (blue) and after application of XE991. Tail currents measured during voltage steps to $-55 \mathrm{mV}$ from a holding potential of $-30 \mathrm{mV}$ in control ACSF (blue) and after application of XE991 (green). D, Population averages demonstrate that application of XE991 removes the membrane current.

positive control, recordings were made from CA1 pyramidal neurons and results confirm the previous findings, demonstrating that $\mathrm{M}$ current is present in these CA1 pyramidal neurons (Fig. $4 C$ ). During a step from -30 to $-55 \mathrm{mV}$, the rectifying current is completely abolished after application of XE991 $(p<0.01, n=4)$ (Fig. $4 D$ ). It should be noted that the horizontal slices used for recordings in $\mathrm{mEC}$ also contain CA1 pyramidal neurons and the
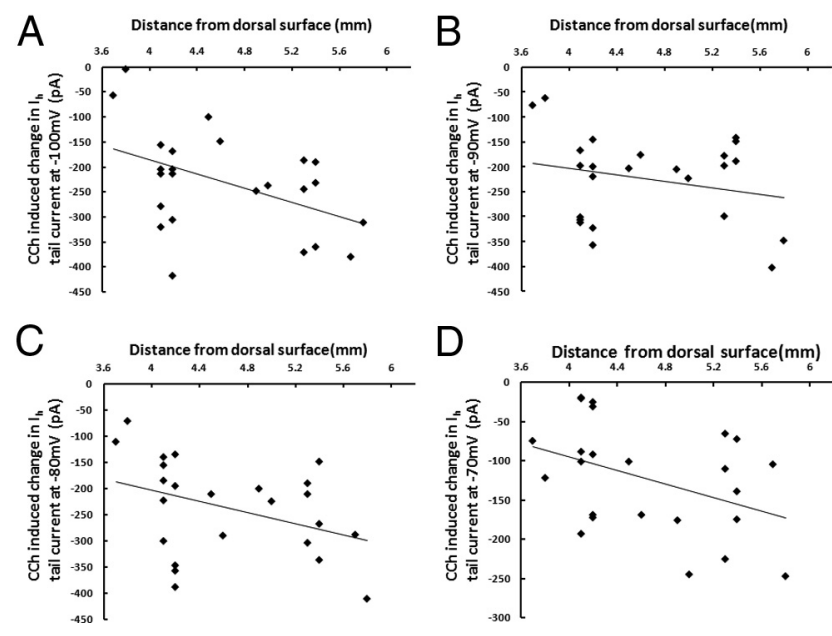

Figure 5. Cholinergic modulation of $I_{\mathrm{h}}$ tail current differs along the dorsal-ventral axis of medial entorhinal cortex. $\boldsymbol{A}$, Changes in tail current amplitudes induced by carbachol (CCh) at steps from a holding potential of -100 to $-40 \mathrm{mV}$, plotted against the dorsal-ventral anatomical location of the stellate cell relative to the dorsal surface of the brain. The linear fit demonstrates significantly larger cholinergic modulation of the $I_{\mathrm{h}}$ tail current in ventral $\mathrm{mEC}$ $(p=0.024, n=24)$. $\boldsymbol{B}$, For changes in tail current amplitudes induced by carbachol at steps from -90 to $-40 \mathrm{mV}$, the linear fit demonstrates a trend toward larger cholinergic modulation of the $I_{\mathrm{h}}$ tail current in ventral $\mathrm{mEC}(p=0.268, n=24)$. $C$, Changes in tail current amplitudes measured at steps from -80 to $-40 \mathrm{mV}$ show a significantly larger cholinergic modulation of the $I_{h}$ tail current in ventral $\mathrm{mEC}(p=0.041, n=24)$. $\boldsymbol{D}$, Changes in tail current amplitudes measured at steps from -70 to $-40 \mathrm{mV}$ show a significantly larger cholinergic modulation of the $I_{h}$ tail current in ventral $\mathrm{mEC}(p=0.0467, n=24)$.
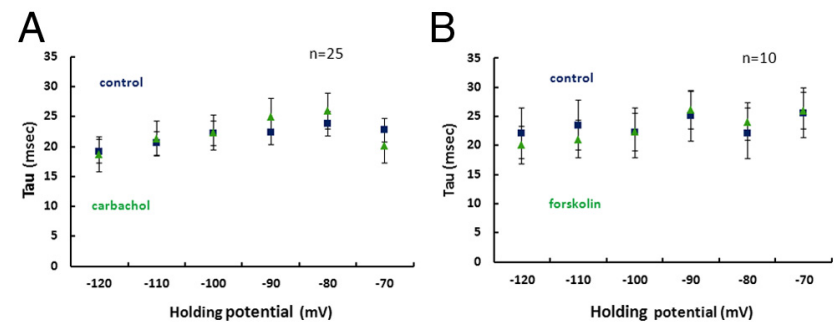

Figure 6. Time course of activation of $I_{h}$. $A$, Time constants of $I_{h}$ activation are shown in control (blue) and in carbachol (green) across hyperpolarized holding potentials ranging from -120 to $-70 \mathrm{mV}$. B, Time constants of $/ \mathrm{h}$ activation are shown in control (blue) and in forskolin (green) across hyperpolarized holding potentials from -120 to $-70 \mathrm{mV}$.

recordings of either SCs in mEC or CA1 pyramidal neurons were made from locations that span the dorsal-ventral extent of the hippocampus and parahippocampal region.

Cholinergic modulation of membrane potential resonance in mEC SCs suggested that modulation occurs differentially along the dorsal to ventral axis of mEC (Heys et al., 2010). Our new data from this current study elucidate the ionic mechanism underlying that previous result by demonstrating that $I_{\mathrm{h}}$ modulation occurs at significantly higher levels in ventral mEC (Fig. 5). Figure $5 A-D$ depicts the change in $I_{\mathrm{h}}$ tail current amplitude after application of $\mathrm{CCh}$, measured at a holding potential of $-40 \mathrm{mV}$ after steps back from $-100,-90,-80$, and $-70 \mathrm{mV}$.

The time constant of $I_{\mathrm{h}}$ activation was measured by fitting a single exponential to the membrane current generated during hyperpolarizing steps from a holding potential of $-40 \mathrm{mV}$. In contrast to studies using heterologous expression of $\mathrm{HCN} 1$, $\mathrm{HCN} 2$, and muscarinic acetylcholine receptors, which have shown a cholinergic-dependent 1.5- to 2-fold slowing of the $I_{\mathrm{h}}$ time constant (Pian et al., 2006, 2007), we found no significant 
A

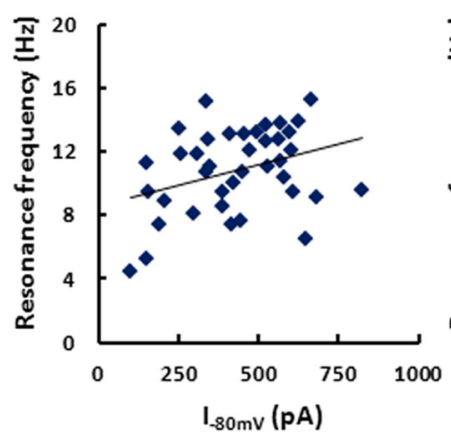

$\mathrm{E}$

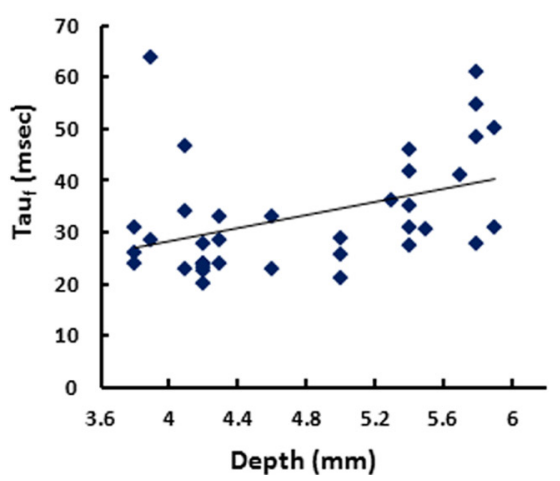

B

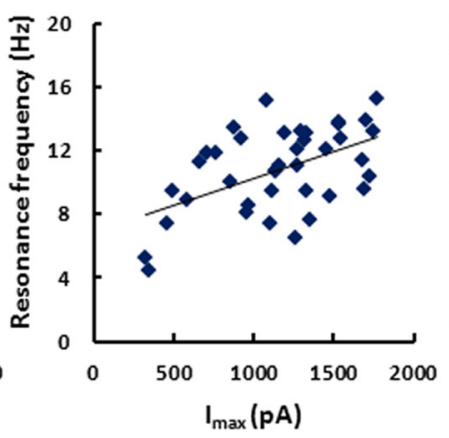

$\mathrm{F}$

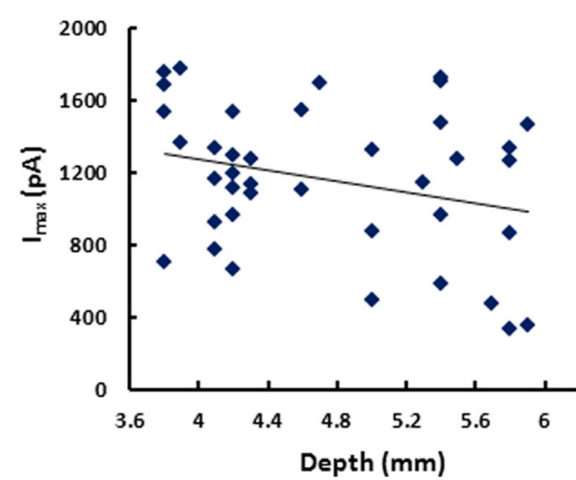

$\mathrm{D}$

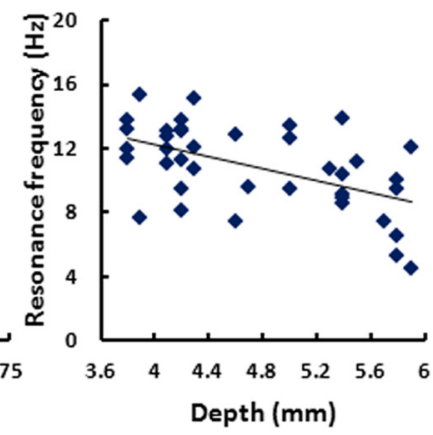

G

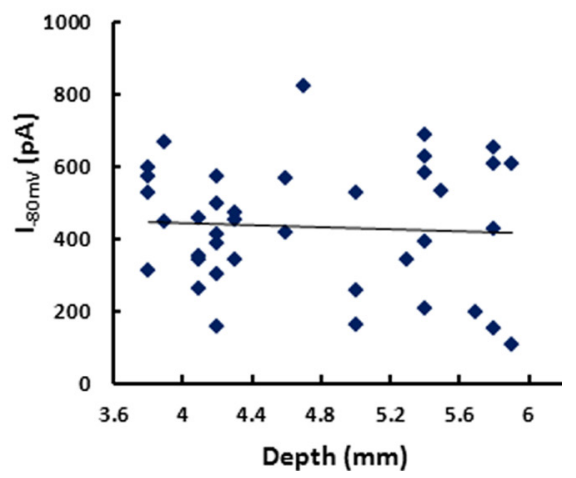

Figure 7. Analysis of the baseline electrophysiological properties of stellate cells. $A$, The magnitude of $I_{\mathrm{h}}$ tail current measured after voltage steps from -80 to $-40 \mathrm{mV}$ demonstrates a significant positive relationship with membrane potential resonance frequency $(p=0.0354, n=39) \cdot \boldsymbol{B}, I_{\mathrm{h}}$ tail current measured at voltage steps from -120 to $-40 \mathrm{mV}$ demonstrates a significant positive relationship with membrane potential resonance frequency $(p=0.0007, n=39)$. C, The time constant of $I_{h}$ activation measured at voltage steps from -80 to $-40 \mathrm{mV}$ demonstrates a significant positive relationship with membrane potential resonance frequency $(p=0.0001, n=36)$. $D$, The membrane resonance frequency decreases significantly from dorsal to ventral $\mathrm{mEC}$ $(p=0.0117, n=36)$. $\boldsymbol{E}$, The time constants of $/ \mathrm{h}$ activation increases significantly from dorsal to ventral $\mathrm{mEC}(p=0.0117, n=36) . \boldsymbol{F}$, The maximum $/ \mathrm{h}$ tail current measure at voltage steps from -120 to $-40 \mathrm{mV}$ shows a trend toward larger amplitude in dorsal $\mathrm{mEC}$, but this does not reach the criteria for statistical significance $(p=0.081, n=39)$. G, There is no significant relationship between $I_{\mathrm{h}}$ tail current amplitude and dorsal-ventral location for tail currents measured at $-80 \mathrm{mV}(p=0.7197, n=39)$.

change in the time course of $I_{\mathrm{h}}$ activation after application of CCh at all membrane potentials measured (Fig. $6 \mathrm{~A}$ ). Similarly, there was no significant change in the $I_{\mathrm{h}}$ activation time constant after application of forskolin (Fig. 6B).

Our results demonstrate that magnitude of $I_{\mathrm{h}}$ can be both upregulated and downregulated through neuromodulation. To correlate the physiological properties of $I_{\mathrm{h}}$ to the characteristic membrane potential resonance present in SCs, we performed voltage-clamp and current-clamp recordings in the same neurons. The $\mathrm{h}$ current tail current amplitude after steps to -80 and $-120 \mathrm{mV}$, where $I_{\mathrm{h}}$ is fully activated, correlates positively which membrane potential resonance frequency $(p<0.05, n=39$; $p<$ $0.01, n=39$ ) (Fig. $7 A, B)$. These results suggest that the cholinergic-dependent decrease in $I_{\mathrm{h}}$ could be responsible for the cholinergic-dependent decrease in membrane potential resonance frequency shown in Heys et al. (2010). These data have important implications for grid cell properties, which will be discussed below. While the results do not show a neuromodulatory effect upon the time constant of $I_{\mathrm{h}}$, the data do show a clear negative relationship between the $h$ current time constant and the membrane potential resonance frequency $(p<0.01, n=36)$ (Fig. 7C). Consistent with findings from Giocomo et al. (2007) and Giocomo and Hasselmo (2008), we found that resonance frequency and the $I_{\mathrm{h}}$ activation time constant differed significantly along the dorsal to ventral axis of $\mathrm{mEC}$ (Fig. $7 D, E$ ). The linear fit to the data demonstrates that, at a membrane potential of $-75 \mathrm{mV}$, the resonance frequency moves from a mean of 12.6
$\mathrm{Hz}$ at the most dorsal aspect of mEC to $8.4 \mathrm{~Hz}$ at the most ventral location in mEC. However, the change in amplitude of $I_{\mathrm{h}}$ along the dorsal-ventral axis is less clear. Similar to Garden et al. (2008), the tail current protocol measured at steps from -120 to $-40 \mathrm{mV}$ suggests a trend toward larger $I_{\mathrm{h}}$ amplitude in neurons at more dorsal locations of $\mathrm{mEC}$, but the trend does not reach criteria for statistical significance ( $p=0.081, n=39$ ) (Fig. 7F). Furthermore, at steps from more depolarized potentials to -40 $\mathrm{mV}$, the distribution of $I_{\mathrm{h}}$ amplitude appears to be uniform along the dorsal-ventral axis ( $p=0.7197, n=39)$ (Fig. $7 G)$.

\section{Discussion}

We have used whole-cell voltage-clamp recordings to isolate and pharmacologically modulate $I_{\mathrm{h}}$, manipulating important neuromodulatory pathways. Our data show that activation of muscarinic cholinergic receptors causes a decrease in the amplitude of the $h$ current and hyperpolarizing shift of the $h$ current activation curve. In contrast, our data also show that activation of the cAMP pathway with forskolin causes an increase in the amplitude and a depolarizing shift in the $h$ current activation curve. Our previous research demonstrates that activation of muscarinic cholinergic receptors produces a decrease in the membrane potential resonance frequency (Heys et al., 2010). The results from the present study resolve questions of which particular ionic mechanism(s) is responsible for this decrease in resonance. In particular, $I_{\mathrm{h}}$ amplitude, and not the time course of $I_{\mathrm{h}}$ or $I_{\mathrm{m}}$, is responsible for the cholinergic-dependent decrease in membrane potential reso- 
nance observed in mEC layer II SCs. Furthermore, the larger cholinergic-dependent decrease in membrane potential resonance frequency in ventral SCs is likely to be caused by the larger cholinergic-dependent decrease in $I_{\mathrm{h}}$ shown in ventral SCs. Since there is an increase in the $\mathrm{I}_{\mathrm{h}}$ activation time constant along the dorsal-ventral axis of $\mathrm{mEC}$ and no change in the time course of $I_{\mathrm{h}}$ activation due to cholinergic modulation, it can be argued that the $I_{\mathrm{h}}$ time constant is likely to be responsible for the systematic decrease in membrane potential resonance frequency along the dorsal-ventral axis in baseline conditions, whereas differential cholinergic modulation of $I_{\mathrm{h}}$ amplitude could be responsible for the differential modulation of membrane potential resonance along the dorsal-ventral axis.

In addition to testing the specific effects of key neuromodulators upon $I_{\mathrm{h}}$, we have tested the hypothesis that there must be distinct neuromodulatory pathways able to both upregulate and downregulate $I_{\mathrm{h}}$ in the medial entorhinal cortex. The results demonstrate that pathways which upregulate cAMP are able shift the $\mathrm{h}$ current activation curve significantly toward more depolarized potentials. In contrast, activation of mAChRs shifts the activation curve in the opposite direction toward more hyperpolarized potentials. Seminal work in the hippocampus demonstrates that release of norepinephrine and subsequent activation of $\beta$-adrenergic receptors increases levels of cAMP (Palmer et al., 1973; Madison and Nicoll, 1986). Furthermore, there is evidence of projections from the locus ceruleus to the entorhinal cortex (Insausti et al., 1987) and evidence of norepinephrine release in the entorhinal cortex that is significantly decreased through ablation of locus ceruleus (Fallon et al., 1978). Collectively, these findings suggest that cAMP-dependent upregulation of $I_{\mathrm{h}}$ in stellate cells could be occurring through noradrenergic release and $\beta$-adrenergic receptor activation.

Based upon previous studies using heterologous expression of $\mathrm{HCN} 1$ and $\mathrm{HCN} 2$ subunits to form functional h current channels and along with expression of mAChRs, we expected to observe a cholinergic-dependent increase in the $\mathrm{h}$ current time constant (Pian et al., 2007). This lack of any significant mAChRdependent change in the $\mathrm{h}$ current time constant in mEC SCs demonstrates the difficulty in predicting modulatory effects across distinct cell types and highlights the need to investigate each case individually. The recent discovery of the PEX5R/Trip8b regulatory subunit demonstrates that cAMP and adrenergic modulation of the $I_{\mathrm{h}}$ activation is reduced when PEX5R/Trip8b is coexpressed with h current channels (Zolles et al., 2009). As such, the lack of a cholinergic effect upon the time course of $I_{\mathrm{h}}$ activation in SCs shown in the present study might be explained by coexpression of PEX5R/Trip8b with $h$ current channels in stellate cells.

The h current appears to play a significant role in regulating the neuronal properties of stellate cells in entorhinal cortex layer II. Using sag percentage as an indicator of the magnitude of $I_{\mathrm{h}}$, the $\mathrm{h}$ current expressed in SCs is nearly twice as large the $\mathrm{h}$ current measured in all other known principal neurons in the entorhinal cortex (Klink and Alonso, 1993; Hamam et al., 2000, 2002; Tahvildari and Alonso, 2005). $I_{\mathrm{h}}$ also plays a significant role in determining steady-state cellular electrophysiology, such as the resting potential and input resistance, and in determining the dynamic responses, such as AHP, membrane potential oscillations, membrane potential resonance, and temporal integration (Dickson et al., 2000; Erchova et al., 2004; Fransén et al., 2004; Garden et al., 2008). Recent in vivo data have further underscored the importance of this current by demonstrating $I_{\mathrm{h}}$-dependent changes in grid field spacing (Giocomo et al., 2011). The resonance proper- ties mediated by h current in stellate cells may contribute to the influence of theta rhythm oscillations on grid cell firing in the entorhinal cortex. Recent work has shown that reductions of theta rhythm oscillations in entorhinal cortex during infusions of muscimol or lidocaine in the medial septum are associated with a loss of grid cell spatial periodicity (Brandon et al., 2011; Koenig et al., 2011). Together with previous studies, this work demonstrates that detailed study of $I_{\mathrm{h}}$ is required for understanding the function of mEC SCs.

The results from this paper have particularly important implications for understanding grid cell function because several lines of research suggest that neuromodulation of $I_{\mathrm{h}}$ may control grid field spacing and grid field size. In the study conducted by Giocomo and colleagues (2011), unit recordings from awake behaving mice show that HCN1 knock-out mice have significantly larger grid field spacing and grid field size compared with wildtype mice. Work in slice experiments demonstrates that knockout of the HCN1 subunit produces an approximately twofold decrease in the $I_{\mathrm{h}}$ tail current amplitude at $-75 \mathrm{mV}$ (Nolan et al., 2007). In addition, grid field spacing and grid field size increase as rodents explore novel environments and return to baseline spacing levels after repeated explorations of the same environment (Barry et al., 2009). Related to this finding, it is thought that levels of acetylcholine increase in the hippocampus during presentation of novel stimuli (Acquas et al., 1996). More recently, it has been demonstrated, using amperometry, that levels of acetylcholine increase during tail pinch of anesthetized rats (Zhang et al., 2010). Together this collection of studies and the data presented in this study suggest that the cholinergic-dependent decrease in $I_{\mathrm{h}}$ occurs as animals explore novel environments and that this decrease in the amplitude of $I_{\mathrm{h}}$ may be responsible for the observed expansion of the grid field spacing and grid field size in the novel environment.

A number of studies have shown that blockade of muscarinic cholinergic receptors with scopolamine impairs memory encoding in a range of different tasks (Aigner and Mishkin, 1986; Ghoneim and Mewaldt, 1977; for review, see Barry et al., 2012), including spatial memory tasks in rats (Whishaw, 1985; Buresova et al., 1986; McGurk et al., 1988; Blokland et al., 1992). The role in learning and memory appears to involve the entorhinal cortex, as shown with selective lesions of cholinergic innervation of the entorhinal cortex (McGaughy et al., 2005) and selective infusions of scopolamine into entorhinal cortex (Esclassan et al., 2009). Understanding the detailed cellular effects of muscarinic modulation on $\mathrm{h}$ current in neurons of the medial entorhinal cortex will help us understand how cellular effects of muscarinic modulation may be important for spatial memory function.

\section{References}

Acquas E, Wilson C, Fibiger HC (1996) Conditioned and unconditioned stimuli increase frontal cortical and hippocampal acetylcholine release: effects of novelty, habituation and fear. J Neurosci 16:3089-3096.

Aigner TG, Mishkin M (1986) The effects of physostigmine and scopolamine on recognition memory in monkeys. Behav Neural Biol 45:81-87.

Alonso AA, Llinás RR (1989) Subthreshold $\mathrm{Na}+$-dependent theta-like rhythmicity in stellate cells of entorhinal cortex layer II. Nature 342:175-177.

Barry C, O'Keefe J, Burgess N (2009) Effect of novelty on grid cell firing. Soc Neurosci Abstr 35:101-124.

Barry C, Heys JG, Hasselmo ME (2012) Possible role of acetylcholine in regulating spatial novelty effects on theta rhythm and grid cells. Front Neural Circuits 6:5.

Blokland A, Honig W, Raaijmakers WG (1992) Effects of introhippocampal scopolamine injections in a repeated spatial acquisition task in the rat. Psychopharmacology 109:373-376. 
Brandon MP, Bogaard AR, Libby CP, Connerney MA, Gupta K, Hasselmo ME (2011) Reduction of theta rhythm dissociates grid cell spatial periodicity from directional tuning. Science 332:595-599.

Buresova O, Bolhuis JJ, Bures J (1986) Differential effects of cholinergic blockade on performance of rats in the water tank navigation task and in a radial water maze. J Behav Neurosci 100:476-482.

Dickson CT, Magistretti J, Shalinsky MH, Fransén E, Hasselmo ME, Alonso A (2000) Properties and role of Ih in the pacing of subthreshold oscillations in entorhinal cortex layer II neurons. J Neurophysiol 83:2562-2579.

DiFrancesco D, Tortora P (1991) Direct activation of cardia pacemaker channels by intracellular cyclic AMP. Nature 351:145-147.

Erchova I, Kreck G, Heinemann U, Herz AV (2004) Dynamics of rat entorhinal cortex layer II and III cells: characteristics of membrane potential resonance at rest predict oscillation properties near threshold. J Physiol 560:89-110.

Esclassan F, Coutureau E, Di Scala G, Marchand AR (2009) A cholinergicdependent role for the entorhinal cortex in trace fear conditioning. J Neurosci 29:8087-8093.

Fallon JH, Koziell DA, Moore RY (1978) Catecholamine innervation of the basal forebrain. II. Amygdala, suprarhinal cortex and entorhinal cortex. J Comp Neurol 180:509-532.

Fransén E, Alonso AA, Dickson CT, Magistretti J, Hasselmo ME (2004) Ionic mechanisms in the generation of subthreshold oscillations and action potential clustering in entorhinal layer II stellate neurons. Hippocampus 14:368-384.

Fyhn M, Hafting T, Treves A, Moser MB, Moser EI (2007) Hippocampal remapping and grid realignment in entorhinal cortex. Nature 446:190-194.

Garden DL, Dodson PD, O’Donnell, White MD, Nolan MF (2008) Tuning of Synaptic Integration in medial entorhinal cortex to organization of the grid cell firing fields. Neuron 60:875-889.

Ghoneim MM, Mewaldt SP (1977) Studies on human memory: The interactions of diazepam, scopolamine, and physostigmine. Psychopharmacology 52:1-6.

Giocomo LM, Hasselmo ME (2008) Time constants of h current in layer II stellate cells differ along dorsal to ventral axis of medial entorhinal cortex. J Neurosci 28:9414-9425.

Giocomo LM, Hasselmo ME (2009) Knock-out of HCN1 subunit flattens dorsal-ventral frequency gradient of medial entorhinal neurons in adult mice. J Neurosci 29:7625-7630.

Giocomo LM, Zilli EA, Fransén E, Hasselmo ME (2007) Temporal frequency of subthreshold oscillations scales with entorhinal grid cell field spacing. Science 315:1719-1722.

Giocomo LM, Hussaini SA, Zheng F, Kandel ER, Moser MB, Moser EI (2011) Grid cells use HCN1 channels for spatial scaling. Cell 147:1159-1170.

Haas JS, White JA (2002) Frequency selectivity of layer II stellate cells in medial entorhinal cortex. J Neurophysiol 88:2422-2429.

Hafting T, Fyhn M, Molden S, Moser MB, Moser EI (2005) Microstructure of a spatial map in the entorhinal cortex. Nature 436:801-806.

Halliwell JV, Adams PR (1982) Voltage-clamp analysis of muscarinic excitation in hippocampal neurons. Brain Res 250:71-92.

Hamam BN, Kennedy TE, Alonso A, Amaral DG (2000) Morphological and electrophysiological characteristics of layer $\mathrm{V}$ neurons of the rat medial entorhinal cortex. J Comp Neurol 418:457-472.

Hamam BN, Amaral DG, Alonso AA (2002) Morphological and electrophysiological characteristics of layer $\mathrm{V}$ neurons of the rat lateral entorhinal cortex. J Comp Neurol 451:45-61.

Heys JG, Giocomo LM, Hasselmo ME (2010) Cholinergic modulation of the resonance properties of stellate cells in layer II of medial entorhinal cortex. J Neurophysiol 104:258-270.
Hu H, Vervaeke K, Storm JF (2002) Two forms of electrical resonance at theta frequencies, generated by $\mathrm{M}$-current, $\mathrm{h}$-current and persistent $\mathrm{Na}+$ current rat hippocampal pyramidal cells. J Physiol 545:783-805.

Insausti R, Amaral DG, Cowan WM (1987) The entorhinal cortex of the monkey: III. Subcortical afferents. J Comp Neurol: 264:396-408.

Klink R, Alonso A (1997) Ionic mechanisms of muscarinic depolarization in entorhinal cortex layer II neurons. J Neurophysiol 77:1813-1828.

Koenig J, Linder AN, Leutgeb JK, Leutgeb S (2011) The spatial periodicity of grid cells is not sustained during reduced theta oscillations. Science 332:592-595.

Ma L, Shalinsky MH, Alonso A, Dickson CT (2007) Effects of serotonin on the intrinsic membrane properties of layer II medial entorhinal cortex neurons. Hippocampus 17:114-129.

Madison DV, Nicoll RA (1986) Cyclic adenosine 3', 5'-monophosphate mediates $\beta$-receptor actions of noradrenaline in rat hippocampal pyramidal cells. J Physiology 372:245-259.

McGaughy J, Koene RA, Eichenbaum H, Hasselmo ME (2005) Cholinergic deafferentation of the entorhinal cortex in rats impairs encoding of novel but not familiar stimuli in a delayed nonmatch-to-sample task. J Neurosci 25:10273-10281.

McGurk SR, Levin ED, Butcher LL (1988) Cholinergic-dopaminergic interactions in radial-arm maze performance. Behav Neural Biol 49:234-239.

Moser EI, Moser MB (2008) A metric for space. Hippocampus 18:1142-1156.

Neher E (1992) Correction for liquid junction potential in patch clamp experiments. Methods Enzymol 207:123-131.

Nolan MF, Dudman JT, Dodson PD, Santoro B (2007) HCN1 channels control resting and active integrative properties of stellate cells from layer II of the entorhinal cortex. J Neurosci 27:12440-12451.

Palmer GC, Sulser F, Robison GA (1973) Effects of neurohumoral and adrenergic agents on cyclic AMP levels in various areas of the rat brain in vitro. Neuropharmacology 12:327-337.

Pian P, Bucchi A, Robinson RB, Siegelbaum SA (2006) Regulation of gating and rundown of HCN hyperpolarization-activated channels by exogenous and endogenous PIP2. J Gen Physiol 128:593-604.

Pian P, Bucchi A, DeCostanzo A, Robinson RB, Siegelbaum SA (2007) Modulation of cyclic nucleotide-regulated HCN channels by PIP2 and receptors coupled to phospholipase C. Pflügers Arch 445:125-145.

Rosenkranz JA, Johnston D (2006) Dopaminergic regulation of neuronal excitability through modulation of $I_{\mathrm{h}}$ in layer $\mathrm{V}$ entorhinal cortex. J Neurosci 26:3229-3244.

Storm JF (1990) Potassium currents in hippocampal pyramidal cells. Prog Brain Res 83:161-187.

Tahvildari B, Alonso A (2005) Morphological and electrophysiological properties of lateral entorhinal cortex layer II and II principal neurons. J Comp Neurol 491:123-140.

Whishaw IQ (1985) Cholinergic receptor blockade in the rat impairs locale but not taxon strategies for place navigation in a swimming pool. Behav Neurosci 99:979-1005.

Yoshida M, Alonso A (2007) Cell-type-specific modulation of intrinsic firing properties and subthreshold membrane oscillations by the $\mathrm{M}(\mathrm{Kv} 7)$-current in neurons of the entorhinal cortex. J Neurophysiol 98:2779-2794.

Zhang H, Lin SC, Nicolelis MA (2010) Spatiotemporal coupling between hippocampal acetylcholine release and theta oscillations in vivo. J Neurosci 30:13431-13440.

Zolles G, Wenzel D, Bildl W, Schulte U, Hofmann A, Müller CS, Thumfart JO, Vlachos A, Deller T, Pfeifer A, Fleischmann BK, Roeper J, Fakler B, Klöcker N (2009) Associations with the auxiliar subunit PEX5R/Trip8b controls responsiveness of $\mathrm{HCN}$ channels to cAMP and adrendergic stimulations. Neuron 62:814-825. 